University of Nebraska - Lincoln

DigitalCommons@University of Nebraska - Lincoln

Ralph Skomski Publications

Research Papers in Physics and Astronomy

2-2012

Berry phase from a randomly fluctuating magnetic field

Ralph Skomski

University of Nebraska-Lincoln, rskomski2@unl.edu

Follow this and additional works at: https://digitalcommons.unl.edu/physicsskomski

Part of the Physics Commons

Skomski, Ralph, "Berry phase from a randomly fluctuating magnetic field" (2012). Ralph Skomski Publications. 69.

https://digitalcommons.unl.edu/physicsskomski/69

This Article is brought to you for free and open access by the Research Papers in Physics and Astronomy at DigitalCommons@University of Nebraska - Lincoln. It has been accepted for inclusion in Ralph Skomski Publications by an authorized administrator of DigitalCommons@University of Nebraska - Lincoln. 


\title{
Berry phase from a randomly fluctuating magnetic field
}

\author{
R. Skomski ${ }^{\text {a) }}$ \\ Department of Physics and Astronomy and Nebraska Center for Materials and Nanoscience, \\ University of Nebraska, Lincoln, Nebraska 68588, USA
}

(Presented 31 October 2011; received 23 September 2011; accepted 5 October 2011; published online 6 February 2012)

\begin{abstract}
The quantum-mechanical Berry phase due to a randomly fluctuating magnetic field is calculated by exploiting an analogous random-walk problem in polymer physics. The phase depends on the time correlations of the magnetic field, despite the adiabatic nature of the Berry phase. A probability distribution for the phase is obtained, and how this phase could affect the magnetotransport in granular magnetic nanostructures is briefly discussed. (C) 2012 American Institute of Physics. [doi:10.1063/1.3670063]
\end{abstract}

\section{INTRODUCTION}

Since its discovery, the Berry phase ${ }^{1}$ has revolutionized quantum mechanics and perpetrated many areas of physics, including magnetism. It means that the wave function acquires a geometrical phase, $|\psi>\rightarrow \exp (\mathrm{i} \gamma)| \psi>$ that is unrelated to the dynamical phase, $\exp (-\mathcal{H} t / \hbar)$, from the time-dependent Schrödinger equation. The Berry phase is important for the understanding of the orbital magnetic moment of itinerant electrons, for quantum entanglement, and for a number of magnetotransport phenomena, such as the anomalous Hall effect. ${ }^{2}$ In fact, the Berry phase of a spin- $1 / 2$ particle in an adiabatically changing magnetic field is one of the first examples of this phenomenon. ${ }^{1}$ The phase is well-defined when the field forms a closed loop and is essentially equal to the solid angle enclosed by the field, that is, to the corresponding area on the unit sphere. For spin-1/2 particles, the Berry phase obeys the simple relation, ${ }^{1,6}$

$$
\gamma=\Omega / 2
$$

where $\Omega$ is the solid angle of the loop. For nanoparticles that can be described as rigidly exchange-coupled macrospins of size, $S$, this equation changes to $\gamma=S \Omega{ }^{6}$

In this paper, we determine the Berry phase caused by a randomly fluctuating magnetic field. Due to the adiabatic nature of the phase, the field must be slow compared to any ongoing quantum-mechanical processes, which is typically the case for meso- and macroscopic magnetic fields. To determine the solid angle enclosed by the magnetic field, we exploit a very similar problem in polymer physics, namely the two-dimensional random-walk with closed loops, which is also known as the problem of topologically constrained polymers. ${ }^{3-5}$

\section{SCIENTIFIC BACKGROUND: ORIGIN OF THE BERRY PHASE}

The Berry phase is created by varying the field angle rather than the field strength, because it is an adiabatic

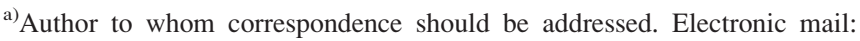
rskomski@neb.rr.com.
}

process and the spin is always parallel to the external magnetic field. Any change in the magnitude of the field yields an ordinary Schrödinger-type dynamical phase, which does not interfere with the Berry phase. ${ }^{1}$

In fact, the Berry phase of a spin in a magnetic field may actually be considered as a "zero-energy" effect. ${ }^{6}$ The external field, $\mathbf{H}(t)$, corresponds to a time-independent adiabatic energy, $E_{\mathrm{o}}=-\mu_{\mathrm{o}} \mu_{\mathrm{B}} \boldsymbol{\sigma} . \mathbf{H}$, so that one can use a simple unitary transformation to adjust the zero of the energy scale and ensure that $E_{\mathrm{o}}=0$. The nontrivial meaning of the corresponding " $\mathcal{H}=0$ " problem is easily seen by considering the action, $S=\int \mathscr{L} \mathrm{dt}$, whose straight minimization corresponds to the system's classical motion, whereas the path integral, $\int \exp (\mathrm{iS} / \hbar) \mathrm{D} x \mathrm{D} p$, yields the quantum-mechanical amplitude. The Lagrangian, $\mathscr{L}$, contains not only the Hamiltonian, $\mathcal{H}$, but also a geometrical contribution describing the phase space. For the linear motion of a particle, $\mathscr{L}=p \mathrm{~d} x / \mathrm{d} t-\mathcal{H}$, however, the "flat" character of the $p-x$ phase space makes the geometrical term uninteresting and the physics is determined by the Hamiltonian. However, the cross-product commutation rules for spins correspond to spin precession and mix the $x, y$, and $z$ spin components, meaning that the geometrical term in $\mathscr{L}$ cannot be neglected. ${ }^{6}$ Figure 1 visualizes this effect in terms of the parallel transport in a curved phase space.

The simplest way to rationalize the Berry phase is to assume a time-independent field magnitude, $H$. In the considered adiabatic limit, this corresponds to a constant Zeeman energy, $E=-\mu_{\mathrm{o}} \mu_{\mathrm{B}} H$, and the previously mentioned unitary transformation ensures that we can use $E=0$. The dynamical phase is, therefore, zero and the Schrödinger equation predicts an unchanged wave function, $|\psi(\mathrm{t})\rangle=|\psi(0)\rangle$. However, this unchanged wave function is contradictory to our starting assumption that the field leads to an adiabatic rotation of the spin, $|\psi(t)\rangle=|\psi(\theta, \varphi)\rangle$. The paradox is solved by the Berry phase, which yields the correct wave function without changing the Hamiltonian.

\section{RANDOM-WALK STATISTICS}

The next step is to map the random magnetic-field direction, shown in Fig. 2, onto a polymer analogy. The use of magnetic models in polymer physics and vice versa has a 


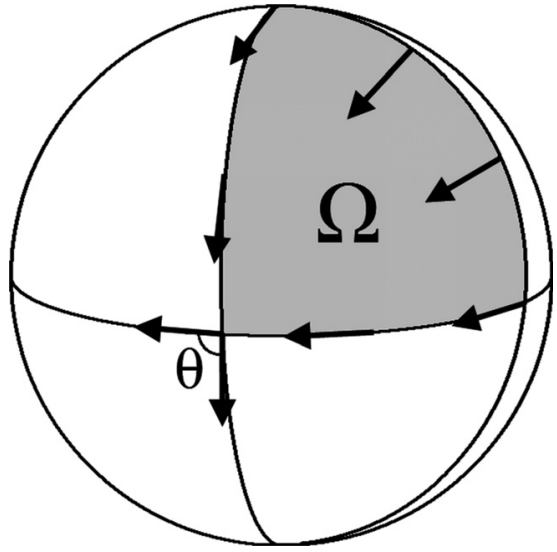

FIG. 1. Parallel transport on a unit sphere. The area, $\Omega$, corresponds to the $S=1$ Berry phase.

long history, such as the use of the $n=0$ vector-spin model to explain phase transitions in polymers. ${ }^{7,8}$ Here we assume that the magnet fluctuates without long-time memory, so that we can map the field variation onto a random-walk diffusion or polymer problem. The details of the short-time field variation are relatively unimportant.

A well-known example in polymer physics is the valence-angle chain, where the $i$ th polymer segment, $\mathbf{l}_{\mathrm{i}}$, is not freely jointed to $\mathbf{l}_{\mathrm{i}-1}$ but confined to a cone defined by the bond angle, $\theta$. In this case, $l_{\mathrm{o}}{ }^{2}=l^{2}(1+\cos \theta) /(1-\cos \theta)$, where $l^{2}=l_{\mathrm{i}}{ }^{2}$. For $\theta=0$, one obtains $l_{\mathrm{o}}=\infty$, corresponding to a stiff and long linear molecule, however, $l_{\mathrm{o}}$ is finite for any nonzero $\theta$, and the macroscopic chain behavior depends only on $l_{\mathrm{o}}$ and no longer explicitly on $\theta$. The Kuhn or statistical segment length, $l_{\mathrm{o}}$, is defined by $\left\langle\mathbf{l}_{\mathbf{i}} \mathbf{l}_{\mathbf{i}+1}\right\rangle=0$ and $\left\langle\mathbf{l}_{\mathbf{i}}{ }^{2}\right\rangle=l_{\mathrm{o}}{ }^{2}$, that is, as the bond length of an equivalent freely jointed polymer chain. This maps the polymer chain onto a random walk, or, alternatively, onto a Markovian diffusion process with $\left\langle\mathbf{R}_{\mathrm{N}}\right\rangle=0$ and $\left\langle\mathbf{R}_{\mathrm{N}}\right\rangle^{2}=N l_{\mathrm{o}}{ }^{2}$, as illustrated in Fig. 3(a).

In the present analogy, we assume that the field fluctuations are small, so that the direction of the field vector remains close to $\mathbf{H}(0)$. Rather than using the field angles, $\theta$ and $\phi$, we can now write,

$$
\mathbf{H}(t)=H(0) \mathbf{e}_{\mathrm{z}}+H_{\mathrm{x}}(t) \mathbf{e}_{\mathrm{x}}+H_{\mathrm{y}}(\mathrm{t}) \mathbf{e}_{\mathrm{y}} .
$$

To describe the randomness, we use the approximation,

$$
\begin{gathered}
H_{\mathrm{x}}(t+\delta t)=H_{\mathrm{x}}(t)+\delta H \mathrm{x} \quad \text { and } \\
H_{\mathrm{y}}(t+\delta t)=H_{\mathrm{y}}(t)+\delta H \mathrm{y},
\end{gathered}
$$

where $\left\langle\delta H_{\mathrm{x}}\right\rangle=\left\langle\delta H_{\mathrm{y}}\right\rangle=0$ and $\left\langle\delta H_{\mathrm{x}}{ }^{2}+\delta H_{\mathrm{y}}{ }^{2}\right\rangle=h_{\mathrm{o}}{ }^{2}$. The trajectory of the magnetization direction, defined in terms of $H_{\mathrm{x}}(t)$ and $H_{\mathrm{y}}(t)$, is analogous to the case of a random-walk polymer chain in two dimensions, and $h_{\mathrm{o}}$ corresponds to Kuhn's segment length, $l_{\mathrm{o}}$. One of the parameters, $h_{\mathrm{o}}$ or $t_{\mathrm{o}}$, may be replaced by the angular velocity, $\eta=h_{\mathrm{o}} / H_{\mathrm{x}} t_{\mathrm{o}}$. Similar to the polymer analogy, the parameters, $t_{\mathrm{o}}$ and $h_{\mathrm{o}}$, include the effect of short-range correlations.

\section{RESULTS AND DISCUSSION}

The magnetization paths of interest for the Berry phase form closed random-walk loops, such as $C_{1}$ and $C_{2}$ in

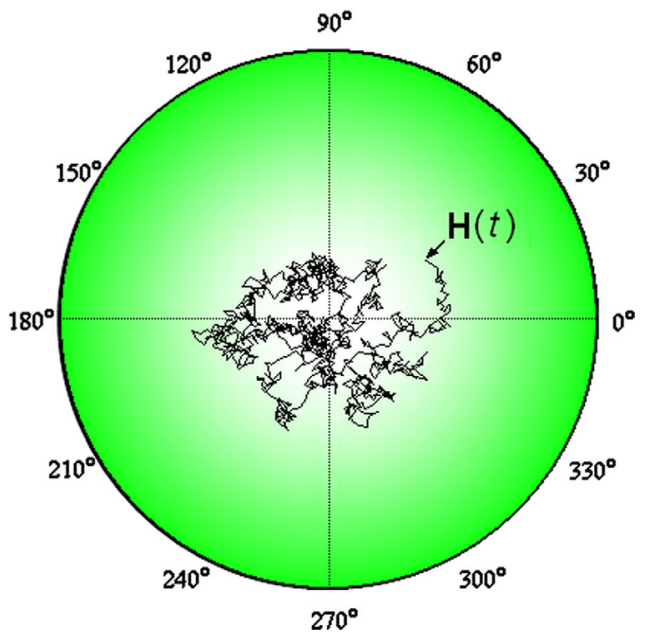

FIG. 2. (Color online) Random-walk description of a fluctuating magnetic field.

Fig. 3(b). The number of these contours is infinite, and any average over these contours has the character of a functional integral,

$$
\langle F\rangle=\int F(C) \mathrm{D} C
$$

Here, the integration includes all closed paths, $C(\mathbf{H}(t))$. In the present case, $F$ is the area in the $H_{\mathrm{x}}-H_{\mathrm{y}}$ plane.

To calculate the functional integral of Eq. (4), we adopt the procedure developed by Khandekar and Wiegel. ${ }^{4}$ The calculation of the area is tedious but yields a transparent

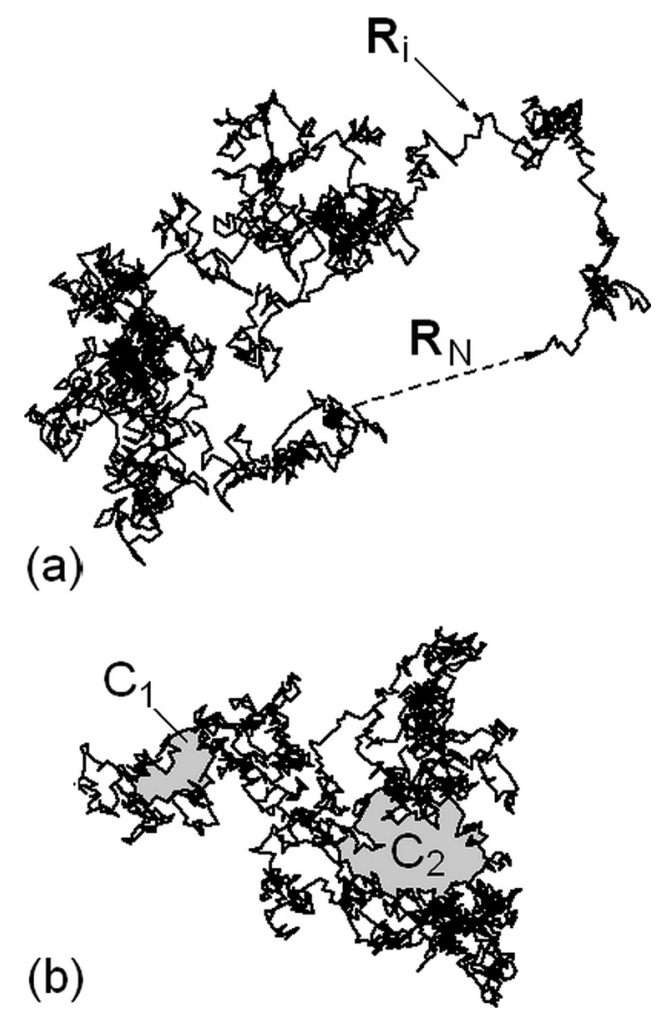

FIG. 3. Random walk: (a) positions of the $i$ th and $N$ th segments, and (b) typical closed loops, $C_{1}$ and $C_{2}$. 


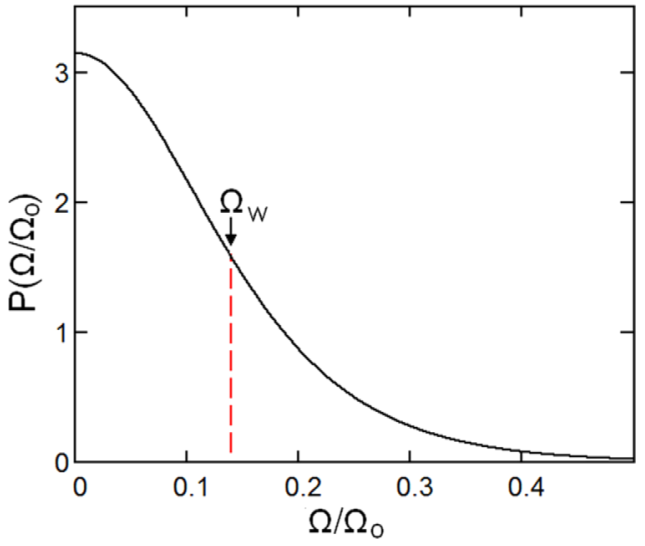

FIG. 4. (Color online) Probability distribution of the normalized Berry phase. The parameter, $\Omega_{\mathrm{o}}$, increases linearly with the waiting time, $t$.

analytical result for the areal probability distribution. ${ }^{4}$ The probability is a function of the ratio, $A / N l_{\mathrm{o}}{ }^{2}$, where $A$ is the considered loop area and $\mathrm{Nl}_{\mathrm{o}}{ }^{2}$ is the average squared end-toend distance. In the magnetic analogy, it is convenient to consider the ratio, $\Omega / \Omega_{\mathrm{o}}$, where,

$$
\Omega_{\mathrm{o}}=\frac{t h_{o}^{2}}{H_{z} t_{\mathrm{o}}},
$$

is some average area corresponding to the writing time, $t$. This yields the probability distribution,

$$
\mathrm{P}(\Omega)=\frac{\pi}{\Omega_{\mathrm{o}} \cosh ^{2} \frac{2 \pi \Omega}{\Omega_{\mathrm{o}}}} .
$$

Figure 4 shows this distribution. Both positive and negative areas are possible, depending on the clockwise or counterclockwise character of the field loops, so that $\langle\Omega\rangle=0$. However, the width, $\Omega_{\mathrm{w}}$, of the distribution, defined by $\Omega_{\mathrm{w}}{ }^{2}=\left\langle\Omega^{2}\right\rangle$ is nonzero, and from Eq. (6) we obtain $\Omega_{\mathrm{w}}=0.144 \Omega_{\mathrm{o}}$.

Here, $\Omega$ can be negative and includes, in general, contributions from subloops with an opposite sense of rotation. This can be seen from the integral used to evaluate the area enclosed by the polymer chain, namely $A=[1 / 2] \int(x \mathrm{~d} y-y \mathrm{~d} x) .{ }^{4}$ In Fig. 4, only the positive half of the distribution is shown, and $\Omega_{\mathrm{w}}$ is marked by the dashed line.

Equation (6) and Fig. 4 become invalid for very long times. This is easily seen by considering that $\Omega$ cannot be larger than $4 \pi$, whereas $\Omega_{\mathrm{o}}$ is proportional to $t$. Physically, the field moves all around the unit sphere, and Eq. (4) can no longer be used. The "angular velocity" $\eta=h_{\mathrm{o}} / H_{\mathrm{z}} t_{\mathrm{o}}$, and the requirement, $H_{\mathrm{x}}{ }^{2}+H_{\mathrm{y}}{ }^{2} \ll H_{\mathrm{o}}{ }^{2}$, yield the condition, $t \ll 1 / \eta^{2} t_{\mathrm{o}}$. Naturally, a small angular velocity, $\eta$, helps to prolong this time, however, a similar effect is achieved by reducing the time step (or correlation time), $t_{\mathrm{o}}$. For fixed $\eta$, this means that rapid changes of the field direction (small $t_{\mathrm{o}}$ ) reduce the enclosed area.

\section{DISCUSSION AND CONCLUSIONS}

It is known that the Berry phase gives rise to quantuminterference effects with far-reaching impact on the magnetotransport, for example, on the anomalous Hall effect. ${ }^{2,10}$ Physically, this involves magnetic noncollinearities and related interaction mechanisms, such as the DzyaloshinskiMoriya interactions and spin-orbit coupling. ${ }^{11}$ A similar effect exists in polycrystalline nanostructures. When an electron travels through such a material, it accumulates a Berry phase by experiencing the rapidly "changing" local magnetic field of the differently oriented magnetic grains. This randomness is of the type described in this paper and affects the propagation of the electron.

So far, we have restricted ourselves to spin-1/2 particles, where $\gamma=\Omega / 2$. As previously mentioned, $\gamma=S \Omega$, where $S$ is the total spin. ${ }^{6}$ Small nanoparticles can be considered as macrospins with $S \gg 1$, so that the Berry phase can assume very high values, even for small field changes. However, this phase is difficult to determine, because the large spin generally implies that $\gamma=2 \pi n+\Delta \gamma(n$ integer and large, $\Delta \gamma<2 \pi)$ and only $\Delta \gamma$ is easily detected by interference experiments.

In conclusion, we have calculated how a slowly varying random magnetic field affects the Berry phase of magnetic particles. The phase exhibits a distribution whose width is determined by the angular velocity of the magnetic field. This introduces a time-dependent aspect into the problem, despite the adiabatic character of the Berry phase. Mechanisms similar to that described in the present paper are operative and possibly important in granular magnetic nanostructures.

\section{ACKNOWLEDGMENTS}

The author is grateful to D. J. Sellmyer and P. Kharel for stimulating discussions. This research is supported by the NSF-MRSEC (Grant No. NSF-DMR-0820521) and NCMN.

${ }^{1}$ M. V. Berry, Proc. R. Soc. London, Ser. A 392, 45 (1984).

${ }^{2}$ D. Xiao, M.-Ch. Chang, and Q. Niu, Rev. Mod. Phys. 82, 1959 (2010).

${ }^{3}$ M. G. Brereton and C. Butler, J. Phys. A 20, 3955 (1987).

${ }^{4}$ D. C. Khandekar and F. W. Wiegel, J. Phys. A 21, L563 (1988).

${ }^{5}$ J. A. Mingo and A. Nica, J. Comb. Theory, Ser. A 84, 55 (1998).

${ }^{6}$ X.-G. Wen, Quantum-Field Theory of Many-Body Systems (Oxford University Press, Oxford, 2004).

${ }^{7}$ P. G. de Gennes, Scaling Concepts in Polymer Physics (Cornell University Press, Ithaca, 1979).

${ }^{8}$ R. Skomski, Simple Models of Magnetism (Oxford University Press, Oxford, 2008).

${ }^{9}$ B. Erman and J. E. Mark, Structures and Properties of Rubberlike Networks (Oxford University Press, Oxford, 1997).

${ }^{10}$ A. G. Aronov and Y. B. Lyanda-Geller, Phys. Rev. Lett. 70, 343 (1993).

${ }^{11}$ R. Skomski, J. Phys.: Condens. Matter 15, R841 (2003). 